\title{
Current singularities in planar magnetic $X$ points of finite compressibility
}

\author{
I. J. D. Craig \\ Department of Mathematics, University of Waikato, Private Bag 3105, Hamilton, New Zealand \\ Yuri E. Litvinenko \\ Institute for the Study of Earth, Oceans, and Space, University of New Hampshire, Durham, \\ New Hampshire 03824-3525
}

(Received 3 August 2004; accepted 3 December 2004; published online 3 February 2005)

\begin{abstract}
The formation of current singularities in nonresistive, line-tied magnetic $X$ points is addressed. It is pointed out that, although gas pressure suppresses the current singularity development when strictly antiparallel, one-dimensional magnetic fields implode, the pressure is likely to be less effective in the more realistic case of two-dimensional magnetic fields. Detailed nonlinear relaxation computations at various levels of compressibility confirm that singularity is present even in the incompressible limit, but its strength, as determined by the amplitude and morphology of the current density, is considerably reduced. The singularity strength is quantified by computing the scalings of the peak current density with resolution. The scalings show that localized current structures can be expected only for negligible gas pressures. The numerical results imply that the inclusion of gas pressure effectively stalls fast magnetic reconnection in line-tied $X$-point geometries. (C) 2005 American Institute of Physics. [DOI: 10.1063/1.1854154]
\end{abstract}

\section{INTRODUCTION}

Magnetic reconnection is a key process in the evolution of astrophysical and laboratory plasmas. One of the most convincing ways to demonstrate the importance of reconnection is to attempt a magnetic merging experiment (real or numerical) in which the plasma resistivity is set to zero. Since the field lines can no longer reconnect, the energy bound up in the global magnetic field eventually manifests itself as a localized current singularity. The strength of the singularity then provides a measure of the energy that can be liberated by resistive merging.

In the limit of strong magnetic field and cold plasma, it is known that disturbances of an initial $X$-point field evolve into strong singularities in the form of $Y$-type current sheets in two dimensions. ${ }^{1,2}$ More general considerations suggest, however, that unless the merging geometry is suitably chosen, the finite gas pressure - or axial components in the magnetic field-inhibits the formation of the singularity. ${ }^{3}$ In this case resistive processes, which require the development of very steep field gradients to be effective, are unlikely to provide significant energy release such as observed in solar flares.

The search for fast reconnection mechanisms that can function in the presence of small but finite gas pressures, say in the solar corona, represents a major research activity. It is surprising, therefore, that the field geometries that allow implosive release of magnetic energy to proceed unhindered by the effects of gas pressure are not well understood. In the present paper we concentrate on the simplest reconnection geometry available to us, that of a line-tied $X$ point in two dimensions. This geometry is known to provide a fast reconnection model in the case of small amplitude displacements of a cold plasma. ${ }^{4-6}$ "Fast" reconnection means that the energy dissipation rate is effectively independent of the nondimensional resistivity (inverse Lundquist number), which can be as low as $\eta \simeq 10^{-14}$ for reconnecting current sheets in the solar corona. It is uncertain, however, to what extent the reconnection rate is quashed by the effects of finite gas pressure. $^{3,7}$

In the present paper we employ magnetic relaxation methods to explore the singular current structures that derive from finite amplitude, nonresistive $X$-point disturbances. Our central aim is to quantify the strength of the singularity as a function of the plasma pressure, as opposed to simply demonstrating the presence of a singularity. ${ }^{8}$ To our knowledge this problem has never been systematically addressed. We also demonstrate how the strength and morphology of the ideal current singularity provides insight into the properties of the resistive current layer that controls the reconnection rate.

The paper is organized as follows. The relationship between the ideal magnetohydrodynamic (MHD) processes and resistive energy dissipation is revisited in Sec. II, where we also discuss in general terms, giving analytical examples, the collapse to singularity. In Sec. III the singular current structures that derive from disturbances of line-tied $X$-point plasmas are explored using a series of relaxation simulations. We summarize in Sec. IV.

\section{THE DEVELOPMENT OF CURRENT SINGULARITIES}

\section{A. Introduction}

It is generally assumed that the form of an ideal current singularity can reflect the structure of resistive current sheets that derive from magnetic merging in weakly resistive plasmas. In particular, if the resistive scaling of the current sheet can be deduced in some manner, say by dimensional or boundary-layer arguments, then the strength of the ideal sin- 
gularity can be used to predict the magnetic reconnection rate. This point is illustrated in the one-dimensional merging problem discussed in Appendix A.

In what follows we model the simplest geometry consistent with magnetic reconnection, namely, a two-dimensional $X$-point geometry in which the field is line tied to rigid, highly conducting, outer boundary. We imagine an equilibrium field, immersed in a uniformly dense plasma that responds dynamically to an initial finite amplitude disturbance. The magnetic field is conveniently described by a dimensionless flux function, $\mathbf{B}=\boldsymbol{\nabla} \psi(x, y, t) \times \hat{\mathbf{z}}$ defined over the region $-1 \leqslant x, y \leqslant 1$, where distances, magnetic fields, and plasma densities are expressed in units of $l_{0}, B_{0}$, and $\rho_{0}$ $=m_{p} n_{0}$, respectively. For typical coronal parameters $l_{0}$ $=10^{9.5} \mathrm{~cm}, B_{0}=10^{2} \mathrm{G}, n_{0}=10^{9} \mathrm{~cm}^{-3}$, the Alfvén speed is $v_{A}=10^{9} \mathrm{~cm} / \mathrm{s}$, and time is conveniently measured in units of $t_{0}=l_{0} / v_{A} \simeq 3 \mathrm{~s}$.

\section{B. Topological and nontopological $X$-point disturbances}

Consider an equilibrium $X$-point $\psi_{E}$ which is excited by the superposition of a disturbance field $\Delta \psi(x, y, t)$, so that initially

$$
\psi(x, y, 0)=\psi_{I}(x, y)=\psi_{E}+\Delta \psi(x, y, 0)
$$

For a current free equilibrium of the form

$$
\psi_{E}(x, y)=\frac{1}{2}\left(x^{2}-y^{2}\right), \rho(x, y, 0)=1,
$$

the initial disturbance field $\Delta \psi(x, y, 0)$ has the effect of raising the $X$-point energy and driving fluid motions. The plasma then evolves dynamically subject to the constraint of line tying on the outer boundary, that is,

$$
\partial_{t} \psi=\mathbf{v}=0 \quad \text { on } S .
$$

We are interested in the properties of the equilibrium solution attained under the conditions of heavy but nonresistive damping. It is important to distinguish between disturbances which alter the equilibrium $X$-point topology and those that do not. If the initial topology is maintained by the disturbance field then any form of damping can reduce the excess energy and return the plasma to its ground state. However, if the disturbance is "reconnective" in the sense that it changes the flux distribution within each lobe of the equilibrium $X$ point, then only magnetic reconnection can dissipate the disturbance energy and recover a current free field.

In the present application we simplify matters by considering only reconnective disturbances $\Delta \psi$ that allow the recovery $\psi_{I} \rightarrow \psi_{E}$ in the case of finite resistivity. Under this restriction viscous relaxation of the initial state $\psi_{I}$ damps out the kinetic energy component but allows the topological component to manifest itself as current singularity if $\eta=0$. The magnetic energy of the final singular state $\psi_{S}$ defines the topological energy of the disturbance. ${ }^{9}$

\section{The MHD equations}

The evolution of the perturbed $X$ point is governed by the continuity, momentum, and induction equations in dimensionless form:

$$
\begin{aligned}
& \frac{D \rho}{D t}=-\rho \boldsymbol{\nabla} \cdot \mathbf{v}, \\
& \rho \frac{D \mathbf{v}}{D t}=-\nabla^{2} \psi \nabla \psi-\nabla P+\mathbf{F}_{\nu}, \\
& \frac{D \psi}{D t}=\eta \nabla^{2} \psi,
\end{aligned}
$$

where $D / D t=\partial / \partial t+\mathbf{v} \cdot \boldsymbol{\nabla}, \mathbf{v}$ is a two-dimensional velocity field, $\rho$ is the density, and $\mathbf{F}_{\nu}$ is a viscous force. The gas pressure $P$ (in units of $\rho_{0} v_{A}^{2}$ ) is defined using the simple polytropic model $P \sim \rho^{\gamma}$. It is convenient to write $\nabla P$ $=\beta^{*} \nabla \rho$, where

$$
\beta^{*}=\frac{1}{2} \gamma \beta
$$

is defined by the plasma beta and the polytropic index.

One possibility for exploring the relaxation $\psi_{I} \rightarrow \psi_{S}$ is simply to simulate the MHD system using strong viscous damping (of the conventional Laplacian form) to dissipate the kinetic energy component of the disturbance. Successively smaller values of the resistivity can then be used to study the collapse to singularity. This approach models the resistive current layer directly but provides, at best, only an approximate "snapshot" of the time-independent singular state $\psi_{s}$. A more satisfactory strategy is to determine the fully relaxed state with $\eta=0$ by using a fictitious "frictional" damping and ignoring the inertia of the plasma. This scheme has the advantage of identically maintaining the field topology while eliminating all wave modes from the plasma. It requires however, the introduction of a fictitious evolution equation for the fluid.

Below we compute and analyze the structure of ideal MHD equilibrium solutions rather than compute the actual evolution of the system toward the equilibrium. Magnetic relaxation methods have been extensively used in studies of the existence and stability of such equilibria ${ }^{10}$ and in studies of the structure of singular equilibria involving current sheets. ${ }^{11,12}$ Although numerical implementations differ, a common feature of the methods is the adoption of a fictitious momentum equation that allows efficient removal of the kinetic energy of the fluid while maintaining the global field topology. Our present purpose is to study the strength and geometrical structure of the relaxed configurations in ideal MHD as a function of gas pressure. ${ }^{13,14}$

\section{The magnetofrictional relaxation}

Given that the properties of the singular equilibrium $\psi_{S}$ is of more concern to us than the dynamics of the evolution $\psi_{I} \rightarrow \psi_{S}$, we exploit the fact that any convenient form of frictional damping can be adopted. By assuming $\mathbf{F}_{\nu}=-\nu \mathbf{v}$ we ensure that frictional damping of the total $X$-point energy occurs at the rate 


$$
\frac{d \varepsilon}{d t}=\mathbf{v} \cdot \mathbf{F}_{\nu}=-\nu \mathbf{v} \cdot \mathbf{v}<0,
$$

where the total energy density is given by

$$
\varepsilon=\frac{1}{2}\left(B^{2}+\rho v^{2}\right)+\frac{P}{\gamma-1} .
$$

Also, by taking $\nu$ large enough, we can eliminate inertial overshoots (MHD oscillations) of the plasma. Note that if we neglect the inertia of the plasma completely, we convert the original MHD equations into a parabolic system of equations in which magnetic and acoustic wave modes are eliminated. ${ }^{15,16}$ The resulting magnetofrictional system compromises neither the final equilibrium nor its linear stability properties.

The relaxation scheme is obtained by setting the left hand side of the momentum equation to zero and taking $\nu$ $=1$ :

$$
\mathbf{v}=-\nabla^{2} \psi \nabla \psi-\beta^{*} \nabla \rho .
$$

This equation, together with the field evolution equation in the limit $\eta=0$

$$
\partial_{t} \psi=-\mathbf{v} \cdot \nabla \psi
$$

and the continuity relation

$$
\partial_{t} \rho=-\nabla \cdot(\rho \mathbf{v}),
$$

allows a convenient Eulerian implementation of the magnetofrictional method.

The numerical scheme that we adopt uses a predictorcorrector time stepping method on a rectangular Cartesian mesh. The use of a planar flux function automatically guarantees the constraint $\boldsymbol{\nabla} \cdot \mathbf{B}=0$ because mixed spatial derivatives of $\psi(x, y, t)$ commute on a rectangular grid. It is important to exclude magnetic reconnection due to numerical resistivity. Reconnection would lead to the transfer of flux through the null point at the origin. Such transfer, however, cannot be achieved by resistive effects because $\eta=0$, and it cannot be achieved by advection because the velocity field is constrained to vanish at the magnetic null by symmetry. Furthermore, the flux across any curve joining the origin to the outer boundary is fixed in our computation because this flux is determined only by the values of $\psi$ at the end points and these values are fixed.

The adopted computational method ensures that the magnetic flux cannot transfer through the null point because of numerical diffusion. Nevertheless, finite numerical resolution is a crucial factor determining the distribution of the computed electric current. The current singularities are manifested numerically by finite current densities that systematically diverge with the resolution. Analytic considerations, such as those presented in Appendix A, suggest that the peak current density should have a power-law dependence on the resolution. This behavior is also suggested by previous numerical solutions representing ideal current sheets. ${ }^{13,14}$ The expected power-law behavior is in fact confirmed by the computations of Sec. III, where we investigate the scaling of the current density with resolution for various gas pressures. It is worth noting that coarse numerical resolution would cause effective diffusion and then the computed structure and magnitude of the electric current would be incorrect. This does not appear to be the case in our simulations, however, because the computed peak current density follows nicely the expected power-law dependence on the resolution. These well-defined scalings show that the numerical solution indeed closely approximates the ideal singularity as the resolution increases.

\section{E. Singular equilibrium solutions}

The result of magnetic relaxation is an equilibrium in which $\mathbf{v}=0$. For this to occur the pressure force in Eq. (9) must act in the $\nabla \psi$ direction, perpendicular to the field lines. This implies $P=P(\psi)$ which leads to the equilibrium condition

$$
\nabla^{2} \psi+P^{\prime}(\psi)=0
$$

provided that the magnetic field is nonvanishing, $|\boldsymbol{\nabla} \psi| \neq 0$. This is simply the familiar Grad-Shafranov equation. It follows that the current density must be constant on the level surfaces of $\psi$. For a polytropic pressure model we also have $\rho=\rho(\psi)$.

Equilibrium structures $\psi_{S}$ deriving from magnetic relaxation experiments have been discussed by various authors. Bajer, ${ }^{11}$ for example, models an incompressible, frictional $X$-point relaxation using flux surface boundary conditions. Evidence in support of a singular final state is provided by scatter plots of current density versus $\psi$, a technique which is also used by Billinghurst, Craig, and Sneyd. ${ }^{12}$

In the present study we seek more quantitative information on the strength of the current singularity by exploring the divergence of the current density against numerical resolution. To motivate this approach we discuss, in Appendix A, the analytic form of the current singularity in the simple case of a one-dimensional magnetic collapse. Figure 1 shows how the relaxed current profiles change dramatically with increasing gas pressure $\left(\beta^{*}=0.01 \rightarrow \beta^{*}=1.0\right)$. The one-dimensional collapse illustrates not only that the current density should increase linearly with resolution in the case of vanishing gas pressure, but also that gas pressure should inhibit the singularity by introducing a length scale $\delta x \simeq \sqrt{\beta^{*}}$ into the current layer. A further interesting feature is that, no matter how small $\beta^{*}$ is, the integrated current can be reduced by no more than a factor of 2 in the frictional relaxation.

Although we can expect gas pressure to stall the magnetic collapse in strictly one-dimensional geometries, more generally the Lorentz force is not irrotational and cannot be balanced by the pressure gradient $\nabla P$. Evidence that singularities develop in nonresistive plasmas is provided in Appendix $B$ where we consider the collapse of a spatially unbounded $X$-point field. This analysis confirms that singularities occur in the form of line discontinuities along the magnetic separatrices. The argument is not definitive, however, since it is based on the assumption of a strong magnetic field, which clearly breaks down at the $X$ point itself. Furthermore, the constraints imposed by a closed ge- 


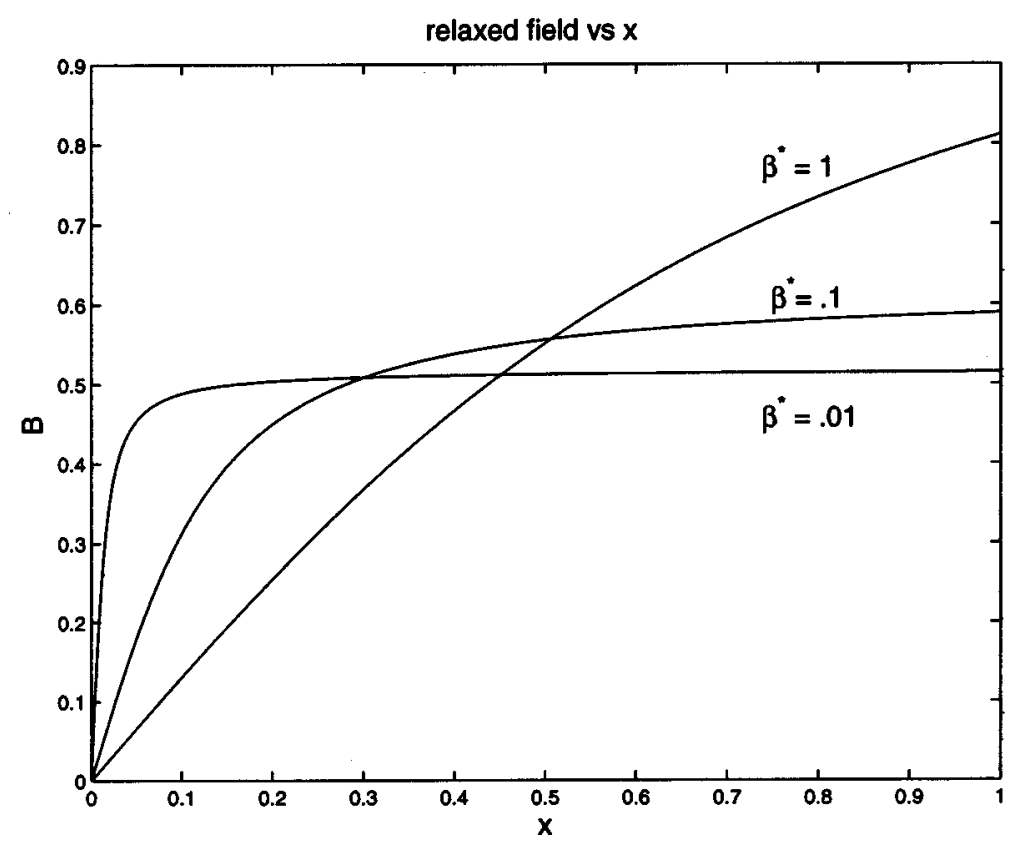

FIG. 1. Relaxed field profiles for the one-dimensional collapse problem with various levels of gas pressure. The steep gradient at the origin signals that a strong current sheet is starting to develop as the gas pressure is reduced.

ometry cannot be represented, nor can line tying be imposed. It appears that only a numerical relaxation treatment can satisfactorily address these issues.

\section{III. $X$-POINT RELAXATION SIMULATIONS}

\section{A. Introduction}

We limit the consideration of the $X$ point collapse to topological disturbances that would allow recovery of the equilibrium $X$ point in the case of resistive relaxation. Specifically, we start with an initial flux function of the form

$$
\psi_{I}(x, y)=\psi_{E}+\Delta \psi_{0}\left(1-x^{2}\right)\left(1-y^{2}\right)
$$

where $\Delta \psi_{0}$ is an arbitrary amplitude and follow the frictional relaxation to steady state. Since this disturbance introduces a finite displacement at the origin which can only be removed by magnetic reconnection, a singular equilibrium is the end result of the frictional relaxation. In the relaxed solutions described below we have taken $\Delta \psi_{0}=0.1$ corresponding to an initial Lorentz force of order unity.

The strength of the magnetic singularity as a function of $\beta^{*}$ is explored by systematically refining the resolution $N$ of the computation, the mesh size being given by $\Delta x=\Delta y$ $\equiv 1 / N$. If a singularity is present then local quantitiesspecifically the current density at the origin-diverge with $N$, even though integrated quantities remain well defined. In particular, since the total current associated with the singularity is determined only by the properties of the initial disturbance we expect that

$$
I_{S} \equiv \int \mathbf{J} \cdot \mathbf{d} \mathbf{A}
$$

should be convergent with resolution.

\section{B. Singular numerical equilibria}

First consider the pressureless relaxation. Since there is no pressure gradient to counterbalance the magnetic forces, the relaxed solution can in principle localize to a scale which is smaller than the mesh size. The computation is therefore halted when gradients comparable to $N$ have developed. Note that when gas pressures are sufficiently large this stopping criterion is not required: the computation, at any resolution, can be simply iterated forward in time until force balance is recovered. In practice, we use the relaxation condition that the maximum net force on the mesh is less than $\simeq 10^{-6}$.

Figure 2 shows the current and density surface plots in the case $\beta^{*}=0$ obtained for $N=100$ points. The strong density gradients in the center of the sheet have, of course, no influence on the structure of the current singularity. But it is clear that the coupling of the density to the gas pressure for $\beta^{*}$ sufficiently large can be expected to strongly modify the magnetic collapse.

Figure 3 shows the current and density surface plots for a typical case of finite pressure $\left(\beta^{*}=1 / 2, N=100\right)$. The central current sheet is now significantly weaker than in the pressureless case, but attached to it are current "wings" that extend almost uniformly along the separatrices of field. The density profile is likewise dramatically modified. The overall density variation is weak, and increases in the east and west quadrants of the $X$-point reflect compressions that compensate for the implosive evacuation of material in the north and south lobes. The density now approximates the equilibrium condition $\rho=\rho(\psi)$ implied by Eq. (12) at all points on the mesh.

\section{Convergence of relaxed equilibria with resolution}

It should be stressed that plots of current density do not, by themselves, provide strong evidence for current singulari- 

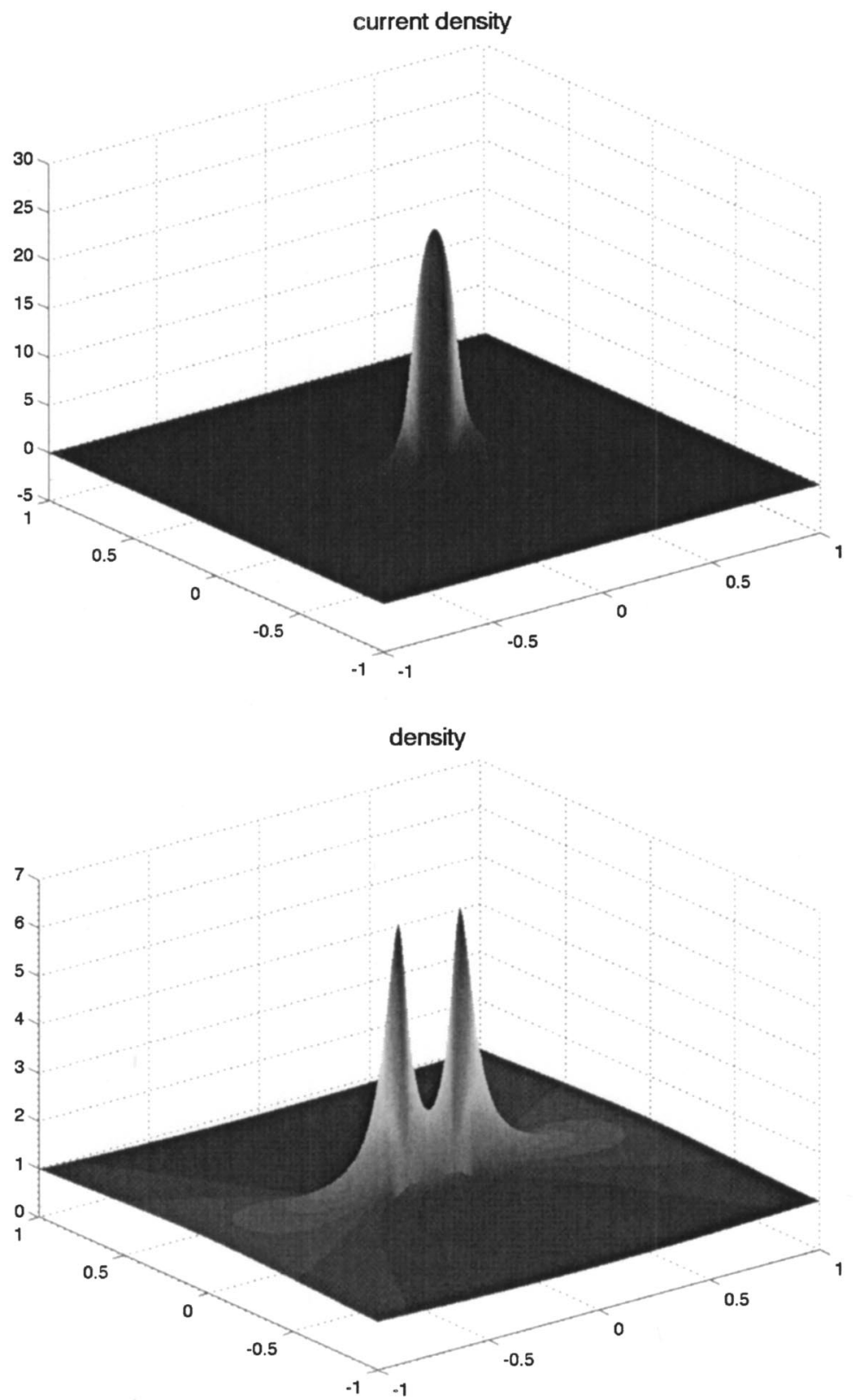

FIG. 2. Relaxed current and density distributions for the case of zero gas pressure $\left(\beta^{*}=0, N=100\right)$. ties. The solutions are generally reasonably smooth with only minor evidence of "ringing" (point to point oscillations due to the under resolution of steep gradients).

To establish singular behavior we examine how the peak current density $J_{S}$ increases with resolution. As an added diagnostic we take the measure $A_{S}(\theta)$, which we define as the area associated with current densities above some fraction $\theta$ of $J_{S}$. Note that $J_{S} A_{S}$ should be approximately invariant with resolution if the current associated with the central peak provides the dominant contribution to the singularity current $I_{S}$.

Consider first the $\beta^{*}=0$ convergence plots shown in Fig. 4. We see that the linear scaling of the current density with $N$ is matched by the fall off in the area measure $A_{S}$ obtained by taking $\theta=1 / 3$. Despite the fivefold increase in resolution, the product $J_{S} A_{S}$ varies by less than $10 \%$, consistent with the conserved quantity $I_{S}$.

Consider now the case of finite pressure, in particular the $\beta^{*}=1 / 2$ scalings of Fig. 5. A salient feature is the weak growth with resolution of $J_{S}$ and the strong decline of the area measure $A_{S}(1 / 3)$. The measured scaling $A_{S} \sim \Delta^{1.8}$ with $\Delta=1 / N$ suggests quasicylindrical current spike, rather than a current sheet. More critically, the variation of the product $A_{S} J_{S}$ clearly contradicts the invariance of $I_{S}$. It follows that, as $N$ increases, the central current "spike" contains an increasingly small fraction of the integrated singularity current.

To obtain a consistent interpretation of the $\beta^{*}=1 / 2$ results we must assume that the wings of the current distribution are the prime contributors to the total singularity current $I_{S}$. Physically, the wing currents should be interpreted in terms of the bifurcation of quasi-one-dimensional current sheet centered at the origin. Measurement of the current density confirms that, despite increases in resolution, the wing current density remains a near constant fraction of $J_{S}$ : for $\beta^{*}=1 / 2$ this contribution is $\approx 15 \%$ when taken along the line 

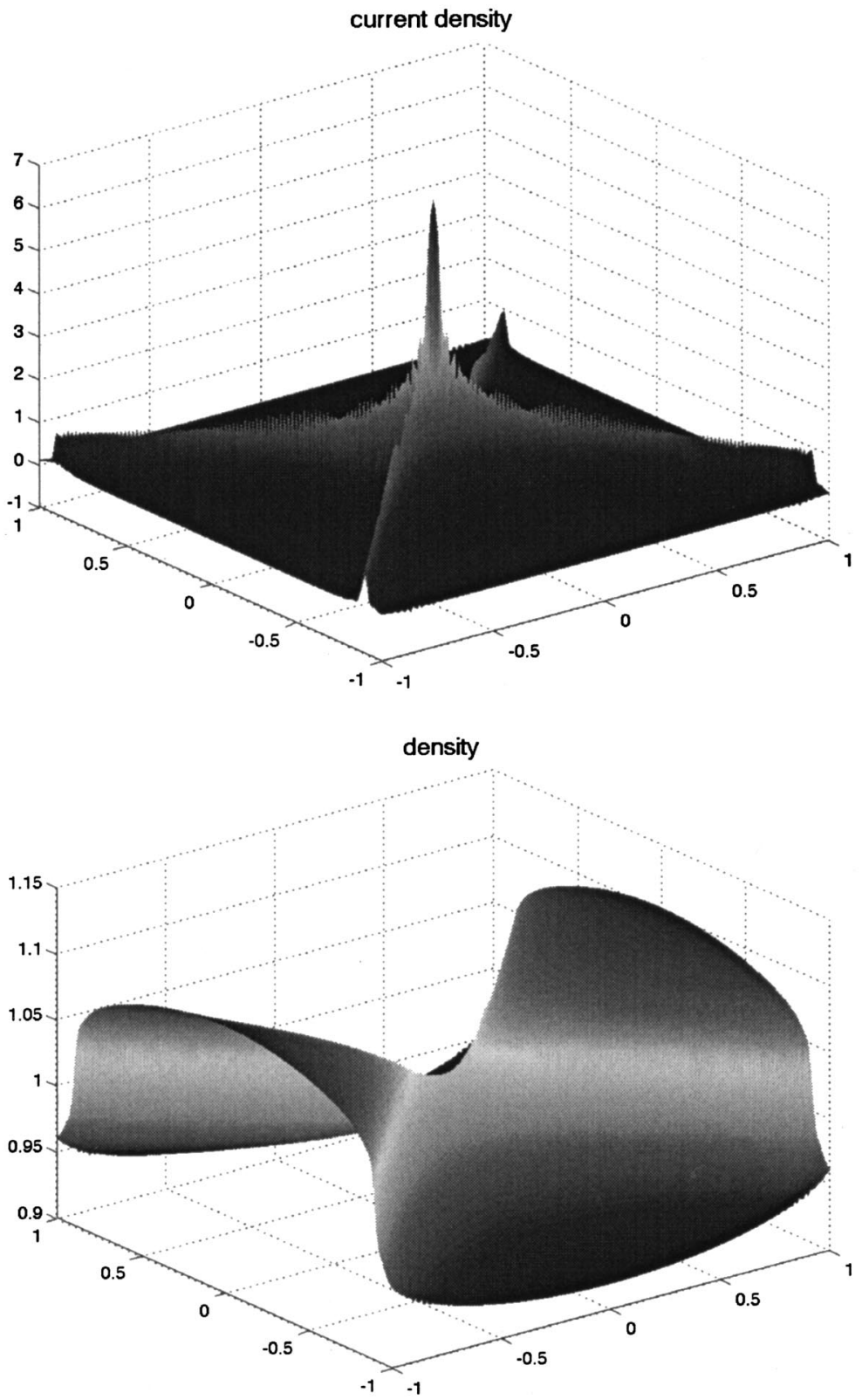

FIG. 3. Relaxed current and density distributions for the case of modest gas pressure $\left(\beta^{*}=1 / 2, N=100\right)$. $x=1 / 2$. By reducing $\theta$ in the area measure $A_{S}$ we pickup the contribution of the wing currents. The $\beta^{*}=1 / 2$ results confirm that with $\theta=1 / 10$ we obtain $A_{S} \sim J_{S}^{-1} \sim \Delta^{0.43}$, a result which now reflects the invariance of the singularity current.

\section{Scalings with plasma pressure}

The results for $\beta^{*}=1 / 2$ are typical in terms of the morphology and the interpretation of the current singularity. Results for a variety of gas pressures confirm that, although the singularity markedly weakens as $\beta^{*}$ increases, it can never be eliminated even for an incompressible plasma. By contrast the integrated current always remains well defined: its initial value of $\approx 1$ is reduced most severely in the pressureless computation to $I_{S}=0.44$ but saturates at around $I_{S} \simeq 0.85$ for the larger pressure amplitudes.

Figure 6 indicates the systematic weakening in the growth of the current density with gas pressure. The fiducial lines indicate logarithmic slopes for the four values of displayed. It follows that $J_{S} \sim \Delta^{-\mu}$ where $\mu=\mu\left(\beta^{*}\right)>0$ decreases with increasing $\beta^{*}$. We also have that $A_{S}(\theta) \sim \Delta^{\mu}$, provided that $\theta$ is chosen small enough to incorporate the dominant current contribution from the wings.

To what extent do these results approximate a truly incompressible plasma? To answer this question we have performed a series of incompressible computations using an independent code. We find that the polytropic model with $\beta^{*}$ $\geqslant 5$ provides current density scalings which closely approximate the incompressible limit.

\section{E. Implications for fast reconnection models}

We now ask whether our results are consistent with fast magnetic reconnection. The collapse of an $X$ point leads to high current densities, so that electric resistivity can no longer be ignored in the vicinity of the neutral point and 


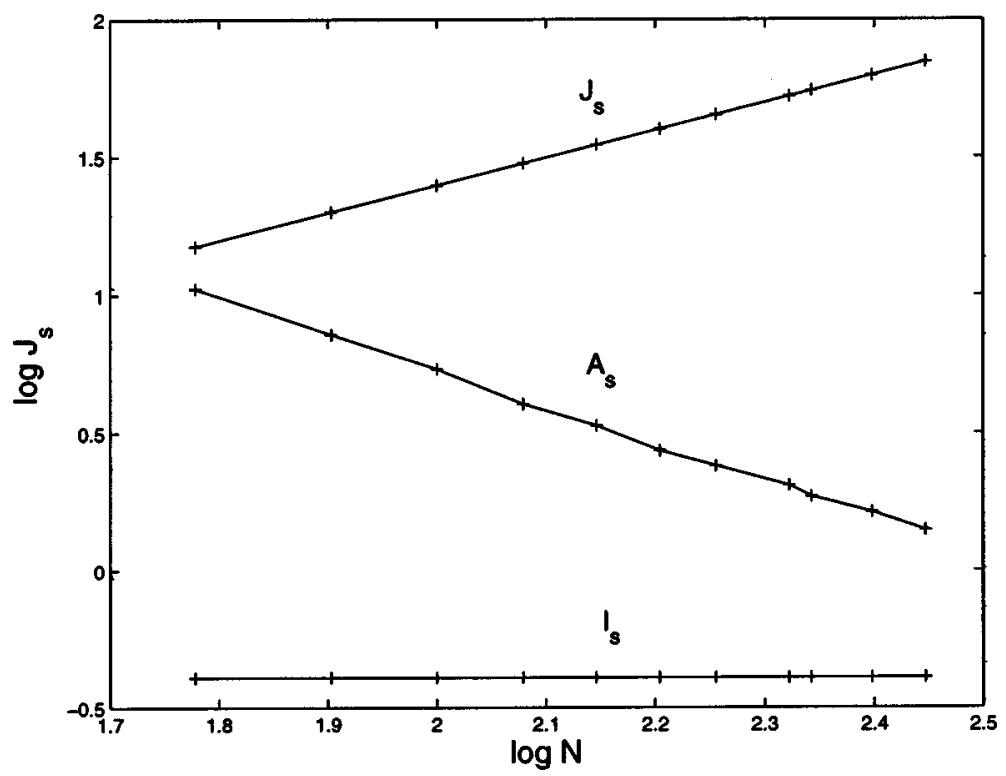

FIG. 4. Scaling of the peak current density $\left(\ln J_{S}\right)$ against resolution $(\ln N)$ for the case of zero pressure. Also shown is a measure $A_{S}(\theta=1 / 3)$ of the area of the current sheet. The product $J_{S} A_{S}$ is almost constant despite the increases in resolution. This product reflects the invariance of the integrated current $I_{S}$ with $N$.

magnetic reconnection takes place. The singularity scalings that we obtained can help us in understanding whether the collapse can be followed by fast reconnection.

For fast reconnection to occur, the reconnection electric field, taken at the neutral point, must be independent of the resistivity. Since $E=\eta J$, we must have $J_{S} \sim \eta^{-1}$. Because our simulations give the scaling of the peak current density $J_{S}$ with the resolution $\Delta$, they also lead to the resistive scaling for the current sheet thickness $\Delta=\Delta(\eta)$, which would be consistent with fast reconnection. Specifically we have seen that $J_{S} \sim \Delta^{-\mu}$ where $\mu<1$ for finite $\beta^{*}$. Hence fast reconnection would imply that $\Delta \sim \eta^{1 / \mu}$. The question is, is this consistency requirement physically plausible?

Consider first the limit $\beta^{*}=0$. The requirement for fast reconnection, $J_{S} \sim \eta^{-1}$, is consistent with a reconnection model in which $\Delta \sim A_{S} \sim \eta$. The morphology of the current singularity shows that merging is achieved via a quasi onedimensional current sheet. Theoretical models of such struc- tures are available and these indeed require sheet thickness to scale as $\eta$ to maintain fast reconnection. ${ }^{3}$ Although this dependence represents the most severe scaling ever simulated numerically or anticipated theoretically, ${ }^{17,18}$ in principle our analysis is consistent with the possibility of fast reconnection in which $J_{S} \sim \eta^{-1}$ in a pressureless plasma.

By contrast, if we naively make the identification $J_{S}$ $\sim \eta^{-1}$ in the case $\beta^{*}=1 / 2$, we obtain $\Delta \sim \eta^{2.3}$, which is not consistent with any reconnection model we are aware of. It is certainly not consistent with dissipation models ${ }^{19}$ based on quasi-one-dimensional current structures extending through each quadrant of the $X$ point, which again require sheet thicknesses that scale as $\eta$. This view is reinforced by noting that fast reconnection requires increasingly severe scalings for larger $\beta^{*}$, in particular $\Delta \sim \eta^{9}$ for $\beta^{*}=5$. We conclude that the resistive scalings required for nonzero $\beta^{*}$ are very difficult to justify on the basis of any known physical model. Our results, therefore, are consistent with fast reconnection

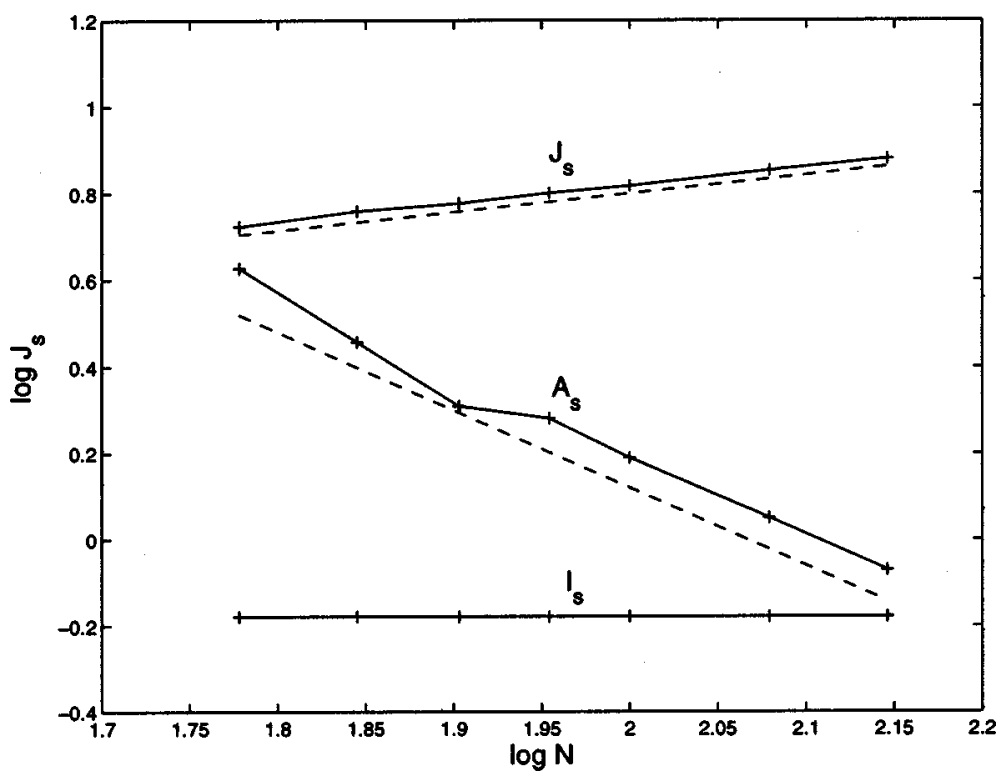

FIG. 5. Scaling of the peak current density $\left(\ln J_{S}\right)$ and the area measure $A_{S}(\theta=1 / 3)$ against resolution $(\ln N)$ for the case $\beta^{*}=1 / 2$. In contrast to the pressureless case the product $J_{S} A_{S}$ no longer reflects the invariance of the integrated current $I_{S}$. The dashed lines show the scalings $J_{S} \sim \Delta^{-0.43}$ and $A_{S} \sim \Delta^{1.8}$. 


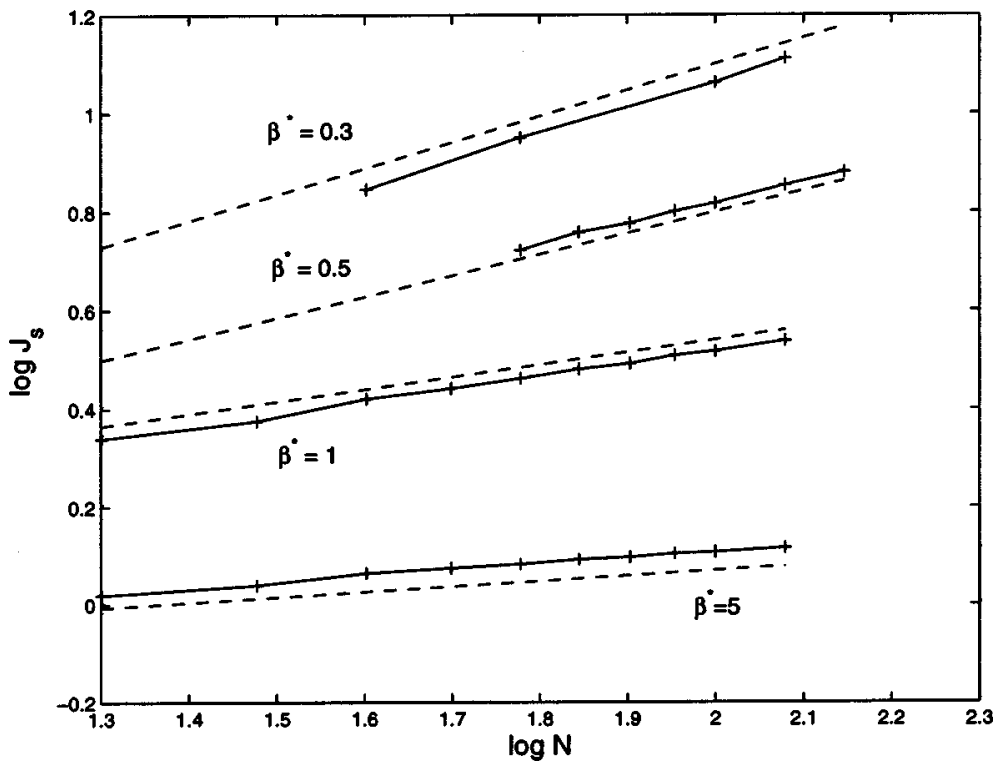

FIG. 6. Scaling of the peak current density $\left(\ln J_{S}\right)$ against resolution $(\ln N)$ for various pressure amplitudes $\beta^{*}$. There is a systematic strengthening of the current singularity as $\beta^{*}$ is reduced. The dotted lines indicate power law slopes of $0.11,0.25,0.43$, and 0.53 . only for $\beta^{*}=0$. In all other cases the current singularity, which weakens progressively with increasing gas pressure, is simply too feeble to allow fast reconnection.

\section{DISCUSSION AND CONCLUSIONS}

We have considered the role of polytropic gas pressure in the nonresistive relaxation of perturbed magnetic $X$ points. A magnetofrictional approach was used to explore the question of whether the gas pressure can suppress the emergence of current singularities for perturbations that alter the initial $X$-point topology. Although the frictional relaxation of the plasma is highly constrained-it takes place within a perfectly conducting rigid boundary to which the field is permanently line tied - singularities invariably emerge, betrayed by a lack of numerical convergence with resolution. The implication is that only in the case of strictly one-dimensional magnetic merging (Appendix A) can gas pressure be expected to stall the collapse.

Gas pressure does, however, greatly modify the structure of the singular equilibrium. In the case of a pressureless plasma, the relaxed solution is a localized current sheet, centered on the magnetic neutral point, in which the peak current density increases systematically with resolution, $J_{S}$ $\sim \Delta^{-1}$. When gas pressure is included, the current singularity is no longer compact: it develops "wings" which extend almost uniformly along the magnetic separatrices. ${ }^{20}$ The scaling of the current density indicates a weak singularity $J_{S}$ $\sim \Delta^{-\mu}$ where $\mu$ is a function of gas pressure: $0<\mu=\mu\left(\beta^{*}\right)$ $\leqslant 1$. This is a different result that quantifies the strength of the singularity as opposed to simply demonstrating its presence.

As the singularity weakens with increases in $\beta^{*}$, the current becomes increasingly concentrated in the separatrix wings. The integrated electric current is always well behaved and depends only weakly on the pressure amplitude. The current varies by less than a factor of 2 over the entire range $\beta^{*}=0$ to $\beta^{*}=\infty$, a result consistent with previous studies. ${ }^{21}$
The ideal singularity scalings severely limit admissible resistive scalings of the current sheet thickness and hence have important implications for magnetic reconnection models. Although the scaling of $J_{S}$ with resolution is not sufficient, in itself, to overrule fast reconnection, our results strongly suggest that, when the geometrical structure of the singularity is taken into account, fast reconnection is possible only in the case $\beta^{*}=0$. The physical interpretation is that for compressible merging in closed, line-tied geometries, the pressure always acts to weaken the singularity and slow down the reconnection rate.

The question remains open as to how these results can be applied to more complicated problems, for example, to reconnection in open or periodic geometries that avoid the restrictions associated with a finite boundary. What can be said is that, in such less constrained geometries where line tying is not imposed, gas pressure is likely to play a less critical role in determining the magnetic merging rate with resistivity. $^{22}$

\section{ACKNOWLEDGMENTS}

This work was supported by the Marsden Fund (Grant No. 02-UOW-050 MIS), by NSF Grant No. ATM-0136718, and by NASA Grant No. NAG5-11797.

\section{APPENDIX A: SINGULARITY IN A ONE-DIMENSIONAL MAGNETIC COLLAPSE}

Let $\mathbf{B}=B_{0}(x) \hat{\mathbf{y}}$ and $\rho_{0}(x)$ be the initial field and density distributions. If $\xi$ is a Lagrangian coordinate that coincides with $x$ initially then flux and mass conservation imply that

$$
\begin{aligned}
& B(\xi) d \xi=B_{0}(x) d x, \\
& \rho(\xi) d \xi=\rho_{0}(x) d x .
\end{aligned}
$$

Here $B(\xi)$ and $\rho(\xi)$ are relaxed distributions that satisfy the pressure balance relation 


$$
B^{2}+\kappa \rho^{2}=A^{2},
$$

where $A$ is constant. Note that to obtain the simplest possible solution we have taken $P=\kappa \rho^{\gamma / 2}$ with $\gamma=2$.

We now specialize to the initial conditions of a magnetic neutral plane in a uniform plasma: $B_{0}=x, \rho_{0}=1$. The pressure balance relation implies

$$
\frac{d \xi}{d x}= \pm \frac{1}{A} \sqrt{\kappa+x^{2}}
$$

which integrates to give

$$
\xi=\frac{1}{2 A}\left\{x \sqrt{\kappa+x^{2}}+\kappa\left[\ln \left(x+\sqrt{\kappa+x^{2}}\right)-\ln \sqrt{\kappa}\right]\right\}
$$

on setting $\xi(0)=0$. $A$ is evaluated by taking $\xi(1)=1$ :

$$
A=\frac{1}{2}\{\sqrt{1+\kappa}+\kappa[\ln (1+\sqrt{1+\kappa})-\ln \sqrt{\kappa}]\} .
$$

Solutions for various levels of compressibility are shown in Fig. 1. The key point is that only in the pressureless case can a singularity develop. Taking $\kappa=0$ we see that $A=1 / 2$ and $\xi=x|x|$ and it follows that the relaxed field and current density can be written as

$$
B=\frac{1}{2}[H(x)-H(-x)], \quad J=\delta(x), \quad|x| \leqslant 1,
$$

where $\boldsymbol{H}(x)$ is the unit step function. The relaxed field is clearly associated with a flux function $\psi(x)=-|x| / 2$ which is nondifferentiable at the origin where the density is infinite:

$$
\rho=\frac{1}{2|x|} .
$$

It is interesting to compare the integrated electric currents $I$ and magnetic energies $M=\int d x d y B^{2} / 2$ in the initial and final configurations. Using Ampère's law to integrate round the boundary $(|x|,|y| \leqslant 1)$ we find that the initial current $(I=4 \rightarrow I=2)$ is reduced by a factor of 2 in the relaxation. This reflects the fact that magnetic energy is lost due to the viscous relaxation of the fluid $(M=2 / 3 \rightarrow M=1 / 2)$.

Consider now the opposite extreme where $\kappa$ is arbitrarily large. In this case $\xi \rightarrow x$ which implies that there is no motion of the plasma. The physical interpretation is that an incompressible plasma can instantly adjust to the implosive tendency of the Lorentz force.

Finally, since we are interested in the numerical representation of current singularities, we should remember that an Eulerian numerical relaxation is limited by the finite mesh spacing $\Delta x=1 / N$. It follows that the maximum current that can be represented numerically is $J_{\max }=B_{s} / \Delta x$ where the sheet field $B_{s}=1 / 2$ according to the argument for $\kappa=\mathbf{0}$. In this case the relaxation scheme will provide a nonconvergent solution in which $J_{\max }$ increases linearly with resolution, $J_{\max } \sim N$, while maintaining a constant integrated current $I$. Note that a Lagrangian relaxation scheme ${ }^{14}$ (which fixes $\rho \Delta x$ on a fluid element) would provide a sharper singularity $J_{\max } \sim N^{2}$.

\section{APPENDIX B: SINGULARITY IN A SPATIALLY UNBOUNDED MAGNETIC $X$ POINT}

It may be argued analytically that a nonzero gas pressure cannot always prevent the formation of ideal MHD singularities in two dimensions. An explicit example is constructed below, which is valid in the approximation of a slowly evolving force-free equilibrium of magnetic field.

Consider first the strong magnetic field approximation for the dynamic evolution of the field, which satisfies both the freezing-in condition and the equation of motion. ${ }^{23,24}$ The strong field approximation describes highly sub-Alfvénic flows, corresponding to a small value of the parameter

$$
\epsilon \equiv v_{0} / v_{A} \ll 1,
$$

where $v_{0}$ is a typical flow speed, and $v_{A}=B_{0} / \sqrt{4 \pi \rho_{0}}$ is the value of the Alfvén speed based on the reference values $B_{0}$ and $\rho_{0}$ of the magnetic field and plasma density. Gas pressure is characterized by a small but non-negligible

$$
\beta \equiv 8 \pi P_{0} / B_{0}^{2} \ll 1,
$$

where $P_{0}$ is the reference pressure. In what follows we assume that $\epsilon^{2}$ is comparable to $\beta$ in order to investigate the gas pressure effects in the formation of MHD singularities. In other words we assume that the evolution time scale $t_{0}$ is sub-Alfvénic but not necessarily subsonic.

Nondimensionalizing the ideal MHD equations, expanding all quantities in power series with respect to $\epsilon^{2}$ and $\beta$, and keeping only zero-order terms leads to the following equations for the zero-order quantities:

$$
\begin{aligned}
& \mathbf{B} \times(\boldsymbol{\nabla} \times \mathbf{B})=0, \quad \boldsymbol{\nabla} \cdot \mathbf{B}=0, \\
& \partial_{t} \mathbf{B}=\delta \boldsymbol{\nabla} \times(\mathbf{v} \times \mathbf{B}), \\
& \partial_{t} \rho+\delta \boldsymbol{\nabla} \cdot(\rho \mathbf{v})=0, \\
& P=\rho^{\gamma} .
\end{aligned}
$$

Here the full energy equation is replaced for simplicity by a polytropic equation of state with $\gamma=$ const. The plasma displacement parameter is defined as

$$
\delta \equiv v_{0} t_{0} / l_{0},
$$

where $l_{0}$ is the reference length scale.

The set of equations above is not complete. One more equation is needed to determine the plasma velocity component along the magnetic field. In the spirit of the standard strong field approximation, we take the scalar product of the full equation of motion and the magnetic field $\mathbf{B}$, divide the result by $\beta$, and obtain another equation that contains only zero-order quantities:

$$
\mathbf{B} \cdot\left(\nabla P+\delta \alpha \rho D_{t} \mathbf{v}\right)=0 .
$$

For convenience we replace $\beta$ by another pressure-related parameter 


$$
\alpha \equiv \frac{2 \epsilon^{2}}{\beta \delta^{2}}=\frac{\rho_{0} l_{0}^{2}}{P_{0} t_{0}^{2}}
$$

and assume that $\alpha \approx 1$, so that the gas pressure effects should eventually come into play. The resulting system of equations describes sub-Alfvénically slow evolution of a force-free magnetic equilibrium.

In general it is almost as difficult to solve the strong field equations as the original MHD equations. It is sufficient, however, to consider the limit of small displacements while searching for the ideal MHD singularities (see Bobrova and Syrovatskii ${ }^{25}$ for the limiting cases $\alpha \rightarrow 0$ and $\left.\alpha \rightarrow \infty\right)$. Assuming the parameter ordering

$$
\epsilon^{2} \ll \delta \ll 1 \approx \alpha,
$$

we expand with respect to $\delta$ the zero-order terms in $\epsilon^{2}$ and $\beta$,

$$
\begin{aligned}
& \mathbf{B}=\mathbf{B}_{0}+\delta \mathbf{B}_{1}+\cdots, \\
& \mathbf{v}=\mathbf{v}_{1}+\cdots, \\
& \rho=1+\delta \rho_{1}+\cdots, \\
& P=1+\delta P_{1}+\cdots,
\end{aligned}
$$

and obtain the following first-order equations:

$$
\begin{aligned}
& \left(\boldsymbol{\nabla} \times \mathbf{B}_{0}\right) \times \mathbf{B}_{1}+\left(\boldsymbol{\nabla} \times \mathbf{B}_{1}\right) \times \mathbf{B}_{0}=0, \\
& \mathbf{B}_{0} \cdot\left(\boldsymbol{\nabla} P_{1}+\alpha \partial_{t t}^{2} \boldsymbol{\xi}\right)=0, \\
& \mathbf{B}_{1}=\boldsymbol{\nabla} \times\left(\boldsymbol{\xi} \times \mathbf{B}_{0}\right), \\
& \rho_{1}=-\nabla \cdot \boldsymbol{\xi}, \\
& P_{1}=\gamma \rho_{1} .
\end{aligned}
$$

These equations can be solved to determine the Lagrangian displacement

$$
\boldsymbol{\xi}=\int_{0}^{t} \mathbf{v}_{1} \mathrm{~d} t
$$

As an illustrative two-dimensional example, consider the dimensionless zero-order magnetic field of a potential magnetic $X$ point:

$$
\mathbf{B}_{0}=(x,-y) .
$$

Although general solutions to the linearized system of MHD equations can be found by standard methods, it is sufficient for our purposes to determine a particular singular solution. We make another simplifying assumption that the electric field $E \sim \mathrm{d}_{t} F$ is uniform in the region, where it is convenient to introduce the function $\mathbf{F}(t)=-\boldsymbol{\xi} \times \mathbf{B}_{0}$.

It is straightforward to verify that a particular exact solution for the displacement is given by

$$
\boldsymbol{\xi}=\left(-\frac{\alpha}{4 \gamma} \ddot{F} y, F \frac{1}{x}+\frac{\alpha}{4 \gamma} \ddot{F} \frac{y^{2}}{x}\right),
$$

where $F^{(4)}(t)=0$. This solution for $\boldsymbol{\xi}(x, y, t)$ gives an example of the ideal MHD singularity in an unbounded region, which is not suppressed by the gas pressure effects.

The formally infinite plasma displacement at $x=0$ corresponds to singularities in the density and pressure perturbations:

$$
\rho_{1}=-\frac{\alpha}{2 \gamma} \ddot{F} \frac{y}{x}, \quad P_{1}=\gamma \rho_{1} .
$$

The pressure force, required to balance the dynamic and magnetic terms in the equation of motion, diverges at $x=0$ as well:

$$
\nabla P_{1}=\frac{\alpha}{2} \ddot{F}\left(\frac{y}{x^{2}},-\frac{1}{x}\right) .
$$

It is worth noting that the singularity in $\boldsymbol{\xi}$ corresponds to a strong flow shear across the $x$ axis, exactly where $\nabla P_{1} \rightarrow \infty$. A limitation of our argument, based on the linearized ideal MHD equations for slow evolution of a force-free magnetic equilibrium, is that the solution becomes invalid at the singularity where the velocity, density, and pressure tend to infinity. We believe, however, that this behavior of the shear and the pressure force is a much more general result in reconnective geometries with nonlocalized current sheets forming at the singularity when resistive effects are taken into account.

${ }^{1}$ R. M. Green, in Proceedings of the IAU Symposium 22 on Stellar and Solar Magnetic Fields, edited by R. Lust (North-Holland, Amsterdam, 1965), p. 398.

${ }^{2}$ S. I. Syrovatskii, Sov. Phys. JETP 33, 933 (1971).

${ }^{3}$ A. N. McClymont and I. J. D. Craig, Astrophys. J. 466, 487 (1996).

${ }^{4}$ I. J. D. Craig and A. N. McClymont, Astrophys. J. 371, L41 (1991).

${ }^{5}$ A. B. Hassam, Astrophys. J. 399, 159 (1992).

${ }^{6}$ I. J. D. Craig and A. N. McClymont, Astrophys. J. 405, 207 (1993).

${ }^{7}$ L. Ofman, P. J. Morrison, and R. S. Steinolfson, Astrophys. J. 417, 748 (1993).

${ }^{8}$ D. Linardatos, J. Fluid Mech. 246, 569 (1993).

${ }^{9}$ I. J. D. Craig, Astron. Astrophys. 283, 331 (1994).

${ }^{10}$ E. R. Priest and T. G. Forbes, Magnetic Reconnection: MHD Theory and Applications (Cambridge University Press, Cambridge, 2000).

${ }^{11}$ K. Bajer, "Flow kinematics and magnetic equilibria," Ph.D. thesis, Cambridge University, 1989.

${ }^{12}$ M. N. Billinghurst, I. J. D. Craig, and A. D. Sneyd, Astron. Astrophys. 279, 589 (1993).

${ }^{13}$ A. W. Longbottom, G. J. Rickard, I. J. D. Craig, and A. D. Sneyd, Astrophys. J. 500, 471 (1998).

${ }^{14}$ F. Ali, and A. D. Sneyd, Sol. Phys. 205, 279 (2002).

${ }^{15}$ I. J. D. Craig, and A. D. Sneid, Astrophys. J. 311, 451 (1986).

${ }^{16}$ W. H. Yang, P. A. Sturrock, and S. K. Antiochos, Astrophys. J. 309, 383 (1986).

${ }^{17}$ H. E. Petschek, in The Physics of Solar Flares, edited by W. N. Hess (NASA, Washington, 1964), p. 425.

${ }^{18}$ T. G. Forbes, J. Plasma Phys. 27, 491 (1982).

${ }^{19}$ I. J. D. Craig and S. M. Henton, Astrophys. J. 434, 192 (1994).

${ }^{20}$ D. Biskamp, Phys. Rep. 237, 179 (1994).

${ }^{21}$ K. V. Brushlinskii, A. M. Zaborov, and S. I. Syrovatskii, Sov. J. Plasma Phys. 6, 165 (1980)

${ }^{22}$ Y. E. Litvinenko and I. J. D. Craig, Sol. Phys. 218, 173 (2003).

${ }^{23}$ S. I. Syrovatskii, Sov. Phys. JETP 23, 754 (1966).

${ }^{24}$ S. I. Syrovatskii, Astrophys. Space Sci. 56, 3 (1978).

${ }^{25}$ N. A. Bobrova and S. I. Syrovatskii, Sol. Phys. 61, 379 (1979). 